\title{
Evidence for phonon skew scattering in the spin Hall effect of platinum
}

\author{
G. V. Karnad, ${ }^{1}$ C. Gorini, ${ }^{2,}{ }^{*}$ K. Lee, ${ }^{1}$ T. Schulz, ${ }^{1}$ R. Lo Conte,,${ }^{1,3}$ A. W. J. Wells, ${ }^{4}$ D.-S. Han,,${ }^{1,5}$ K. Shahbazi, ${ }^{4}$ J.-S. Kim,,${ }^{5, \dagger}$ \\ T. A. Moore, ${ }^{4}$ H. J. M. Swagten, ${ }^{5}$ U. Eckern, ${ }^{6}$ R. Raimondi, ${ }^{7}$ and M. Kläui ${ }^{1,3, \ddagger}$ \\ ${ }^{1}$ Institut für Physik, Johannes Gutenberg-Universität, Staudinger Weg 7, 55128 Mainz, Germany \\ ${ }^{2}$ Institut für Theoretische Physik, Universität Regensburg, 93040 Regensburg, Germany \\ ${ }^{3}$ Graduate School of Excellence “Materials Science in Mainz” (MAINZ), Staudinger Weg 9, 55128 Mainz, Germany \\ ${ }^{4}$ School of Physics and Astronomy, University of Leeds, Leeds LS2 9JT, United Kingdom \\ ${ }^{5}$ Department of Applied Physics, Eindhoven University of Technology, 5600 MB Eindhoven, The Netherlands \\ ${ }^{6}$ Institut für Physik, Universität Augsburg, 86135 Augsburg, Germany \\ ${ }^{7}$ Dipartimento di Matematica e Fisica, Roma Tre University, Via della Vasca Navale 84, 00146 Rome, Italy
}

(Received 6 February 2017; revised manuscript received 30 August 2017; published 26 March 2018)

\begin{abstract}
We measure and analyze the effective spin Hall angle of platinum in the low-residual resistivity regime by second-harmonic measurements of the spin-orbit torques for a multilayer of $\mathrm{Pt}|\mathrm{Co}| \mathrm{AlO}_{x}$. An angular-dependent study of the torques allows us to extract the effective spin Hall angle responsible for the damping-like torque in the system. We observe a strikingly nonmonotonic and reproducible temperature dependence of the torques. This behavior is compatible with recent theoretical predictions which include both intrinsic and extrinsic (impurities and phonons) contributions to the spin Hall effect at finite temperatures.
\end{abstract}

DOI: 10.1103/PhysRevB.97.100405

The demand for high-density and efficient data storage devices has driven the research on magnetic memories. Proposals were mainly based on using spin transfer torques for domain wall motion and magnetization switching [1]. However, spin transfer torque-based devices are plagued by the requirement of high writing current densities which result in deterioration of the material layers and hence limit the device life. A recent demonstration [2] of highly efficient current-induced switching via spin-orbit torques (SOTs) shows promising results that can overcome these limitations [1]. Such devices are made from magnetic multilayers which consist of a few monolayers-thick ferromagnetic (FM) material sandwiched between a normal metal (NM) and an oxide layer.

The injection of an in-plane current through the normal metal has been shown to efficiently manipulate the magnetization [3]. The torques have been attributed to the spin Hall effect (SHE) [3-6] arising from the charge to spin current conversion in the NM and the inverse spin galvanic effect (ISGE) [7-13] arising at the interfaces with the FM.

First, a charge current in the NM underlayer generates via the SHE a spin current flowing towards the FM layer. These spins enter the FM and exert by $s-d$ exchange a predominantly damping-like torque on the FM magnetization [2-4,14]. Second, due to inversion symmetry breaking at the $\mathrm{NM} \mid \mathrm{FM}$ interface, the same charge current gives also rise to an (interfacial) nonequilibrium spin polarization via the ISGE $[10,11,15]$. Such spin polarization is predicted to result predominantly in a field-like torque affecting the FM magnetization. It has actually

\footnotetext{
*cosimo.gorini@physik.uni-regensburg.de

${ }^{\dagger}$ Present address: DGIST Research Center for Emerging Materials, DIGST, Daegu 42988, Republic of Korea.

${ }^{\ddagger}$ klaeui@uni-mainz.de
}

been discussed how the SHE and the ISGE can individually in principle generate both damping-like and field-like torques due to subtler interfacial spin-orbit effects $[16,17]$. Indeed, we will argue later that in our system the SHE is dominating and is responsible for both torques. Irrespective of their origin, whether SHE or ISGE, each torque can be described in terms of the corresponding component of an overall current-induced spin-orbit field acting on the magnetization: a longitudinal component $\mu_{0} H_{D L} \sim \hat{\sigma} \times \hat{m}$, exerting a damping-like torque $\sim \hat{m} \times(\hat{\sigma} \times \hat{m})$, and a transversal one $\mu_{0} H_{F L} \sim \hat{\sigma}$, giving rise to a field-like torque of $\sim \hat{m} \times \hat{\sigma}$. Here $\hat{m}$ is the magnetization direction, and $\hat{\sigma}$ is that of the spin of the (nonequilibrium) SHE and/or ISGE electrons. In our experimental configuration the charge current flows along $\hat{x}$, whereas $\hat{m}=\hat{z}, \hat{\sigma}=\hat{y}$, see Fig. 1 .

Efficient magnetization switching using SOTs has motivated studies of various heavy metals so as to find materials with the highest spin Hall angle (SHA). The SHA characterizes the efficiency of charge-to-spin conversion and is defined as $\theta_{s H}=\sigma_{s H} / \sigma=-\rho_{s H} / \rho$ with $\sigma(\rho)$ and $\sigma_{s H}\left(\rho_{s H}\right)$, respectively, as the charge and spin Hall conductivities (resistivities). There are various mechanisms giving rise to the SHE: extrinsic (side jump [18] and skew scattering [19,20]) and Berry curvature-induced intrinsic ones [21]. In metallic paramagnets it is difficult to identify materials where only one mechanism is at work. It is important to rather understand the dominant mechanism in a particular system in order to optimize it for possible technological applications. Indeed, phonon skew scattering has been recently suggested to play an important role at room temperature [22], and a thorough experimental study of its influence on the SHE is still lacking.

In this Rapid Communication we provide such a study by monitoring the temperature dependence of the SOTs ascribed to the SHE in low-residual resistivity $\operatorname{Pt}(5.3,6.3 \mu \Omega \mathrm{cm})$. Although the observed nonmonotonic behavior is unexpected 


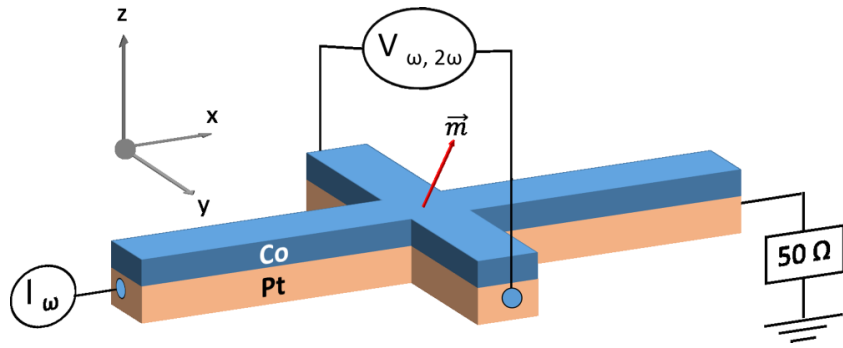

FIG. 1. Schematics of the second-harmonic measurement setup. A charge current $I_{\omega}$ is injected along $\hat{x}$, generating damping-like and field-like effective spin-orbit fields which exert a torque on the magnetization $\hat{m}$. The damping-like torque acts along $\hat{\sigma} \times \hat{m}$, and the field-like one acts along $\hat{\sigma}$. Here $\hat{\sigma}$ is the direction of the currentinduced nonequilibrium spin current (SHE) and density (ISGE).

and cannot be explained following the established phenomenological analysis [23,24], it is compatible with the temperature scaling derived from the microscopic theory [22]. The importance of "ultraclean" samples is further supported by the simpler monotonic behavior observed in high-residual resistivity Ta.

Sample characterization. First, we measure the evolution of the magnetic properties with temperature since the ferromagnetic layers in these stacks are only a few monolayers thick. The temperature dependence of the saturation magnetization in this thin-film structure is measured using superconducting quantum interference device magnetometry. This is plotted in Fig. 2, and in the relevant temperature range a constant value is found. The temperature dependence of the effective anisotropy is studied by measuring the extraordinary Hall voltage in individual Hall crosses during the application of a rotating magnetic field [25]. This angle-dependent variation of Hall voltage (seen in the inset of Fig. 2) when interpreted in terms of the Stoner-Wolfarth model [26] allows us to determine the anisotropy field and hence the effective anisotropy. The effective anisotropy constant is plotted in Fig. 2 and shows no significant variation. These measurements establish that the multilayer has a Curie temperature much larger than $300 \mathrm{~K}$ with no significant changes in magnetic properties in the considered temperature range.

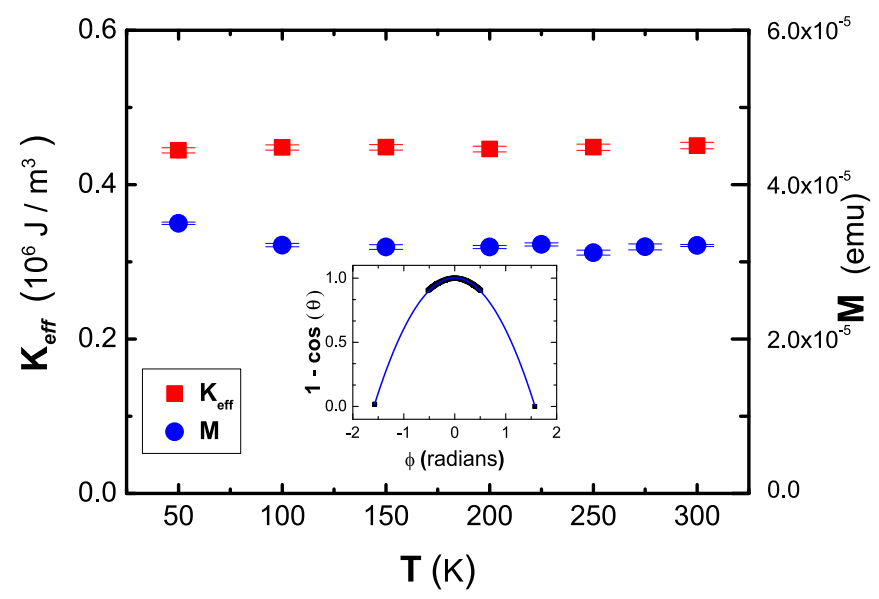

FIG. 2. Temperature dependence of $K_{\text {eff }}$ and $M$.

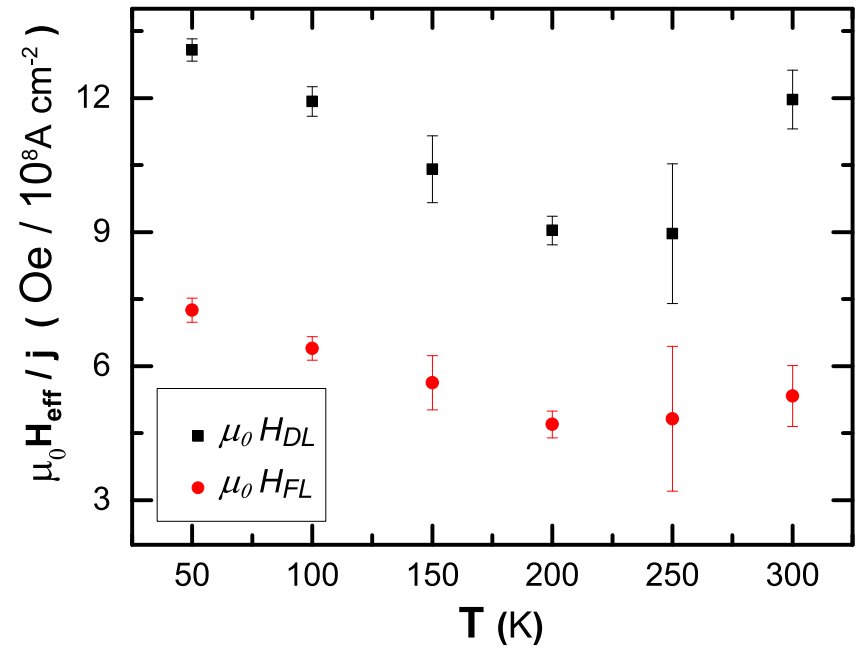

FIG. 3. Temperature dependence of the efficiency of dampinglike $\left(\mu_{0} H_{D L}\right)$ and field-like $\left(\mu_{0} H_{F L}\right)$ effective fields.

Spin-orbit torque measurements. Next, we perform spinorbit torque measurements using the second-harmonics $(2 \omega)$ technique [4,14,27] on patterned Hall bars. The material stack that is used (see the Supplemental Material [28]) is as follows: $\mathrm{Pt}(4.0)|\mathrm{Co}(0.8,1.3)| \mathrm{AlO}_{x}(2.0)$ (all thicknesses in nanometers).

The $2 \omega$ measurements $[4,14,27]$ are performed (see the Supplemental Material [28]) by injecting a low-frequency $(\omega / 2 \pi=13.7-\mathrm{Hz})$ sinusoidal ac signal through the nanowire (along the $x$ axis) while the sample is in a saturated magnetization state (close to the $z$ axis). The effective fields are measured as a function of temperature, and this is shown in Fig. 3. We see a clear nonmonotonic dependence that is distinct from the temperature dependence of the magnetic properties shown in Fig. 2.

The observed temperature dependence of the spin-orbit torques can have various origins, such as: change in magnetic properties, spin-mixing conductance, the SHE, and the ISGE. We individually evaluate all these parameters to pinpoint the origin of the variation of the spin-orbit torques with temperature. First, as already shown, the magnetic properties of our sample are effectively temperature independent [see Fig. 2]. Second, experimental results [29] show that the spin-mixing conductance at the $\mathrm{Pt} \mid \mathrm{Co}$ interface is largely temperature independent as well. We thus attribute any nonmonotonic change in the current-induced effective fields to a variation of the SHE and/or ISGE and the resulting torques exerted.

At this point we make a crucial observation, namely, that damping- and field-like terms change in unison, indicating a common origin for the two torques. This agrees with our previous conclusions [30] of the SHE being the likely cause for both SOTs in $\mathrm{Pt}|\mathrm{Co}| \mathrm{AlO}_{x}$ as well as with results where the SHE was claimed to be the dominant effect for the dampinglike torque in this system [2,3,31]. As mentioned earlier, the generation of both torques via the SHE is due to interfacial spin-orbit effects [17]: Roughly, part of a $\hat{\sigma}$-polarized spin Hall current $J_{S H}$ incoming on the Co layer is lost and turned into a $\hat{\sigma}^{\prime}$-polarized spin current $\delta J_{S H}$ by interfacial spin-orbit coupling. Once in the FM, the (remaining) $J_{S H}$ and $\delta J_{S H}$ 


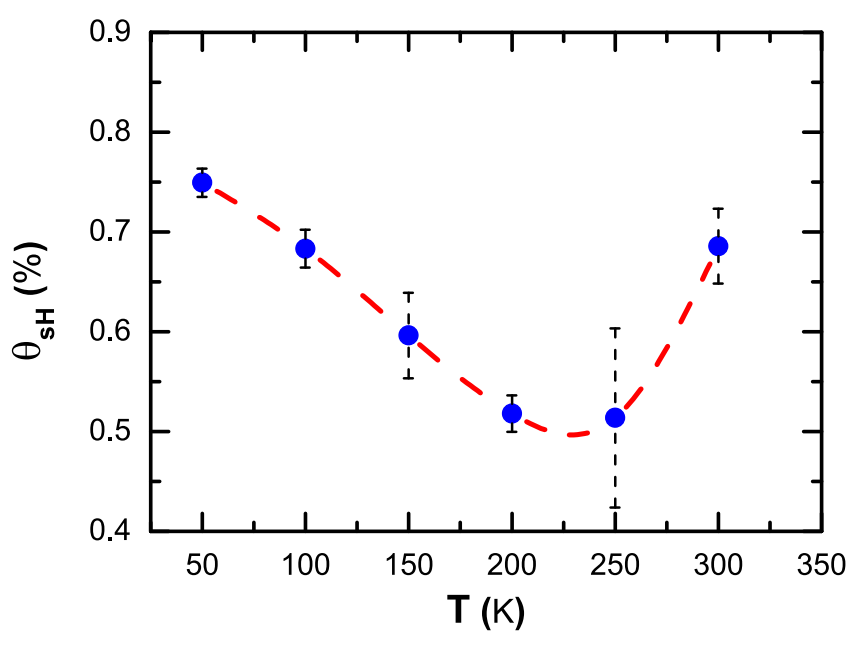

FIG. 4. Temperature dependence of the deduced spin Hall angle (blue dots, connected by a basis spline as a guide to the eye) derived from the damping-like effective field. Practically identical behavior is found in a control sample, see Fig. S4 in the Supplemental Material [28].

exert damping-like and field-like torques, respectively, on the magnetization. Since we cannot quantify the loss $\delta J_{s H}$, we define an effective spin Hall angle assuming that the remaining spin Hall spin current is fully transmitted from $\mathrm{Pt}$ to $\mathrm{Co}$ [2,31-33],

$$
\theta_{s H}=\frac{2 \mu_{0} H_{D L} e M_{s} t_{F M}}{\hbar j_{e}},
$$

where $M_{s}$ is the saturation magnetization, $t_{F M}$ is the ferromagnetic layer thickness, and $j_{e}$ is the injected charge current. We plot in Fig. 4 the temperature evolution of the calculated effective spin Hall angle of Pt.

Theoretical analysis and discussion. We see in Fig. 4 that the effective SHA varies nonmonotonically with temperature. We also observe that there is a significant upturn in its magnitude around $240-250 \mathrm{~K}$. This is a distinct temperature in this system as the spin Hall source in our system Pt is known [34] to have a Debye temperature of $T_{D}=240 \mathrm{~K}$. The Debye temperature $T_{D}$ gives an estimate of the temperature beyond which all phonon modes are excited and behave classically.

The temperature dependence of the SHA provides us with the opportunity to gain insight into the relative magnitude of the different mechanisms responsible for the SHE in the system. We will start by considering both intrinsic and extrinsic (skew scattering) mechanisms: The importance of the former in $4 d$ and $5 d$ transition metals is known [35] and so is the fact that in low-impurity samples the dominant extrinsic contribution comes from skew scattering [6,24,36-39].

We now analyze the temperature dependence of the SHA $[24,40]$ based on the scaling suggested by Tian et al. [23] and the Supplemental Material [28]. The starting point is

$$
\rho_{s H} \approx-\left(\sigma_{s H}^{\text {int }}+\sigma_{s H}^{s s}\right) \rho^{2} \approx-\sigma_{s H}^{\text {int }} \rho^{2}+\rho_{s H}^{s s},
$$

having assumed that skew scattering $\left(\sigma_{s H}^{s s}\right)$ and intrinsic $\left(\sigma_{s H}^{\text {int }}\right)$ contributions act as parallel channels $\sigma_{s H}=\sigma_{s H}^{\text {int }}+\sigma_{s H}^{s s}$ and that $\sigma \gg \sigma_{s H}$. The latter condition implies $\sigma_{s H} \approx \rho_{s H} / \rho^{2}$ with $\rho$ as the longitudinal resistance of the Pt layer and was used in the second passage above. Then it is argued that the skew scattering resistivity scales as $\rho_{s H}^{s s}=\theta_{s H}^{s s, \text { imp }} \rho^{\text {imp }}+\theta_{s H}^{s s, \text { phon }} \rho^{\text {phon }}$ with the spin Hall angle due to phonon skew scattering being temperature independent, whereas $\rho^{\text {imp }}\left(\rho^{\text {phon }}\right)$ represents the resistivity from impurities (phonons). The split $\rho=\rho^{\mathrm{imp}}+$ $\rho^{\text {phon }}$ follows Mathiessen's rule. One thus has

$$
\rho_{s H} \approx-\sigma_{s H}^{\text {int }} \rho^{2}+\theta_{s H}^{s s, \text { imp }} \rho^{\text {imp }}+\theta_{s H}^{s s, \text { phon }}\left(\rho-\rho^{\text {imp }}\right) .
$$

In the lowest-order approximation phonon skew scattering, i.e., the last term on the right-hand side of Eq. (3), is neglected, and $-\rho_{s H}$ can be plotted vs $\rho^{2}$. In this regime, the slope of the linear relation between $\rho_{s H}$ and $\rho^{2}$ describes the intrinsic contribution to the SHE, indicating the sign of the SHA of the material. If this analysis yields inconsistent results, the last term in Eq. (3) is also taken into account [24]. In our case, however, even the full expression (3) is not enough since the relation between $\rho_{s H}$ and $\rho$ is nonmonotonic, see Figs. S5 and S6 of the Supplemental Material [28].

This suggests the need to revisit the established temperature scaling relation assumed for the SHA. The microscopic theory of phonon skew scattering shows indeed that the corresponding SHA is not temperature independent but rather a function of the temperature $\theta_{s H}^{s s, \text { phon }} \rightarrow \theta_{s H}^{s s, \text { phon }}(T)$. As a consequence Eq. (3) is modified to

$$
\rho_{s H} \approx-\sigma_{s H}^{\text {int }} \rho^{2}+\theta_{s H}^{s s, \text { imp }} \rho^{\text {imp }}+\theta_{s H}^{s s, \text { phon }}(T)\left(\rho-\rho^{\text {imp }}\right) .
$$

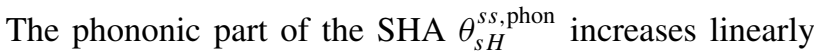
with $T$ at high temperatures ( $\left.T \gtrsim T_{D}\right)$, whereas, clearly, it has to vanish at low temperatures, although its precise behavior at $T<T_{D}$ is not yet known [41]. Its asymptotics can however be used to determine the qualitative behavior of the full SHA in the whole temperature range. An order-of-magnitude estimate for a metallic system yields $\sigma_{s H}^{T_{D}} / \sigma_{s H}^{0} \lesssim 10^{-1}$ with $\sigma_{s H}^{T_{D}}\left(\sigma_{s H}^{0}\right)$ as the spin Hall conductivity at the Debye (zero) temperature [22]. Coupled with the moderate change in the charge conductivity over the same temperature range of $\sigma^{T_{D}} / \sigma^{0} \lesssim 1$, see Fig. S7 of the Supplemental Material [28], one has $\theta_{s H}^{\text {ss, phon }}\left(T \approx T_{D}\right)<$ $\theta_{s H}^{s s, \text { imp }}$. The resulting qualitative behavior shown in Fig. 5 is compatible with the measurements in clean Pt samples. In a clean system the low- $T$ SHE is dominated by skew scattering, i.e., $-\sigma_{s H}^{\text {int }} \rho^{2}(T \rightarrow 0)<\theta_{s H}^{s s \text {,imp }} \rho^{\text {imp }}$. Thus, the SHA should eventually decrease in magnitude from $T=0$ down to a minimum at a temperature $T_{1}$ given by $-\sigma_{s H}^{\text {int }} \rho^{2}\left(T_{1}\right) \approx \theta_{s H}^{s s, \text { imp }} \rho^{\text {imp }}$ and increase afterwards. Furthermore, at a higher temperature of $T_{2} \gtrsim T_{D}$ such that $\theta_{s H}^{s s, \text { phon }}\left(T_{2}\right)\left[\rho\left(T_{2}\right)-\rho^{\text {imp }}\right] \approx \theta_{s H}^{s s \text {,imp }} \rho^{\text {imp }}$ phonon skew scattering would start dominating impurity skew scattering, yielding an asymptotic linear behavior,

$$
\theta_{s H}\left(T \gtrsim T_{2}\right) \sim\left[\sigma_{s H}^{\text {int }}+\sigma_{s H}^{s s, T_{D}}\right] T,
$$

with $\sigma_{s H}^{s s, T_{D}}$ as the saturation value for the phonon skew scattering spin Hall conductivity at $T \gtrsim T_{D}$ [22]. A few comments are in order: (i) The qualitative analysis yields $T_{1} \lesssim T_{D} \lesssim T_{2}$, but we cannot give quantitative estimates of the temperatures $T_{1}, T_{2}$. These require precise knowledge of various systems parameters, notably electron-phonon and phonon-phonon coupling strengths. (ii) The nonmonotonic behavior predicted by Eq. (4) for clean samples does not require phonon skew scattering to be stronger than impurity skew scattering, which 

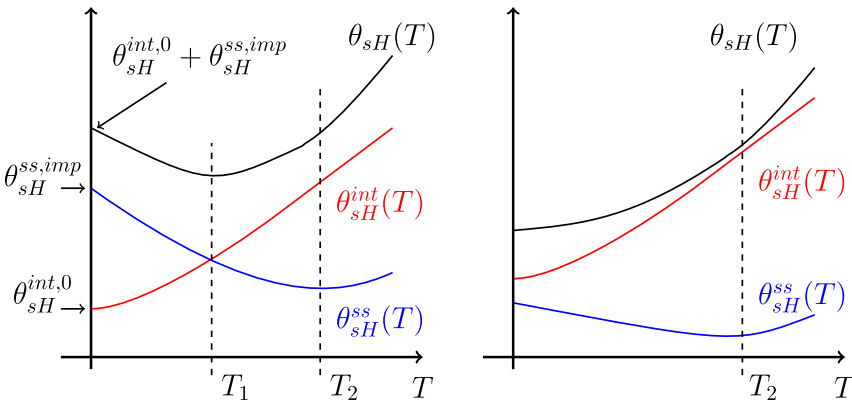

FIG. 5. Sketch of the temperature behavior of the SHA for an ultraclean sample (skew scattering dominated, left panel) and a dirty one (intrinsic dominated, right panel). The sketch is based on Eq. (4) and built from knowledge of the high- and low- $T$ asymptotics, which imply the existence (absence) of a minimum in clean (dirty) systems (see the Supplemental Material [28]). The precise behavior in the intermediate temperature range is however not yet known, nor can accurate values for $T_{1}, T_{2}$ be given since both temperatures depend on various system parameters. An order-of-magnitude estimate for $\mathrm{Pt}$ yields $T_{1} \lesssim T_{D} \lesssim T_{2}$ and suggests that $T_{1}$ should be higher the cleaner the sample. In dirty samples the compensation between intrinsic (red) and skew scattering (blue) curves can give rise to an effectively constant SHA over a broad $T$ range as observed in Ta (for the details see Fig. S4 in the Supplemental Material [28]).

happens only at $T>T_{2}$ : It is enough to recognize that the phonon SHA is itself a function of the temperature $\theta_{s H}^{s s, \text { phon }}=$ $\theta_{s H}^{s s, \text { phon }}(T)$, vanishing at $T=0$ and (linearly) increasing at high temperatures. (iii) Although $\theta_{s H}^{s s, \text { phon }}(T \rightarrow 0)=0$ has to hold, the approach to zero could be nontrivial and affected, e.g., by the interplay between impurity and phonon scattering [42].

The picture changes in dirty systems with strong intrinsic SHE such that $-\sigma_{s H}^{\text {int }} \rho^{2}(T \rightarrow 0)>\theta_{s H}^{s s \text { imp }} \rho^{\text {imp }}$. In this case Eq. (4) predicts a simpler monotonic behavior of the SHA, once more compatible with our measurements in a dirty Ta sample $(137 \mu \Omega \mathrm{cm})$, see Fig. S4 of the Supplemental Material [28]. Ta has a strong intrinsic SHE [2], $T_{D}$ is comparable to that of $\mathrm{Pt}$ [43], and our sample is 20 times as resistive as the Pt sample used. We observe a considerably larger SHA with no minimum, indeed roughly constant in the considered temperature range.

The SHA transition through the intrinsic and extrinsic regimes can be expected due to the low residual resistivity of $\mathrm{Pt}$ $(5.3,6.3 \mu \Omega \mathrm{cm}$ ), as we find (see the Supplemental Material
[28]) here. This is essential because the intrinsic and extrinsic contributions scale differently [24] with the resistivity of the metal. It was recently shown [40] that tuning the conductivity of Pt leads to a transition of the SHE from a "moderately dirty" to a "superclean" regime. This corresponds to a transition from an intrinsic-dominated to an extrinsic-dominated regime. The intrinsic term scales with $\propto \rho^{2}$ and in the low-resistivity limit is dominated by the phonon contribution, which scales roughly with $\propto\left(\rho-\rho^{\mathrm{imp}}\right)$. This could be the reason why other experimental investigations of the spin Hall angle of Pt with higher resistivity have observed the dominance of intrinsic contributions, whereas they have observed dominance of extrinsic contribution in the case of low resistivity $\mathrm{Au}$ [24].

To summarize, we used the second-harmonic technique to measure the spin-orbit torque in $\mathrm{Pt}|\mathrm{Co}| \mathrm{AlO}_{x}$ and $\mathrm{Ta}|\mathrm{CoFeB}| \mathrm{MgO}$.

We deduced the temperature-dependent evolution of the SHA of clean Pt and dirty Ta. The reproducible nonmonotonic behavior observed in Pt cannot be accounted for by standard phenomenology but is compatible with the temperature scaling derived from microscopic phonon skew scattering theory. The monotonic behavior observed in dirty Ta is instead ascribed to the weakness of skew scattering contributions. Our results reveal that extrinsic contribution to the SHE might be the key to understanding the temperature dependence in normal metals as well and thus provides a more comprehensive understanding of the spin Hall angle and the resulting spin-orbit torques in this system.

Acknowledgments. We acknowledge support by the EU (MAGWIRE, Project No. FP7-ICT-2009-5; Marie Curie ITN WALL, Grant No. FP7-PEOPLE-2013-ITN 608031); the DFG (Grants No. KL1811, No. TRR 173, No. TRR 80, No. SFB 1277, and No. SFB 689); the Graduate School of Excellence Materials Science in Mainz (MAINZ) GSC 266; the Research Center of Innovative and Emerging Materials at Johannes Gutenberg University (CINEMA); and the Foundation for Fundamental Research on Matter (FOM), which is part of the Netherlands Organization for Scientific Research (NWO). J.-S.K. acknowledges supports by the Leading Foreign Research Institute Recruitment Program through the NRF funded by the Ministry of Education, Science and Technology (MEST) (Grant No. 2012K1A4A3053565), the DGIST R\&D Program of the Ministry of Science, ICT and Future Planning (Grants No. 17-BT-02 and No. 17-NT-01).
[1] G. Prenat, K. Jabeur, G. Di Pendina, O. Boulle, and G. Gaudin, in Spintronics-Based Computing, edited by W. Zhao and G. Prenat (Springer, Berlin, 2015), pp. 145-157.

[2] L. Liu, C.-F. Pai, Y. Li, H. W. Tseng, D. C. Ralph, and R. A. Buhrman, Science 336, 555 (2012).

[3] L. Liu, O. J. Lee, T. J. Gudmundsen, D. C. Ralph, and R. A. Buhrman, Phys. Rev. Lett. 109, 096602 (2012).

[4] K. Garello, I. M. Miron, C. O. Avci, F. Freimuth, Y. Mokrousov, S. Blügel, S. Auffret, O. Boulle, G. Gaudin, and P. Gambardella, Nat. Nanotechnol. 8, 587 (2013).

[5] G. Y. Guo, S. Murakami, T.-W. Chen, and N. Nagaosa, Phys. Rev. Lett. 100, 096401 (2008).
[6] J. Sinova, S. O. Valenzuela, J. Wunderlich, C. H. Back, and T. Jungwirth, Rev. Mod. Phys. 87, 1213 (2015).

[7] A. G. Aronov and Y. B. Lyanda-Geller, JETP Lett. 50, 431 (1989).

[8] V. M. Edelstein, Solid State Commun. 73, 233 (1990).

[9] S. D. Ganichev, E. L. Ivchenko, V. V. Bel'kov, S. A. Tarasenko, M. Sollinger, D. Weiss, W. Wegscheider, and W. Prettl, Nature (London) 417, 153 (2002).

[10] K. Shen, G. Vignale, and R. Raimondi, Phys. Rev. Lett. 112, 096601 (2014).

[11] J. Borge, C. Gorini, G. Vignale, and R. Raimondi, Phys. Rev. B 89, 245443 (2014). 
[12] I. M. Miron, K. Garello, G. Gaudin, P.-J. Zermatten, M. V. Costache, S. Auffret, S. Bandiera, B. Rodmacq, A. Schuhl, and P. Gambardella, Nature (London) 476, 189 (2011).

[13] S. D. Ganichev, M. Trushin, and J. Schliemann, in Handbook of Spin Transport and Magnetism, 2nd ed., edited by E.Y. Tsymbal and I. Zutic (Chapman and Hall, London, 2016).

[14] M. Hayashi, J. Kim, M. Yamanouchi, and H. Ohno, Phys. Rev. B 89, 144425 (2014).

[15] X. Wang and A. Manchon, Phys. Rev. Lett. 108, 117201 (2012).

[16] P. M. Haney, H.-W. Lee, K.-J. Lee, A. Manchon, and M. D. Stiles, Phys. Rev. B 87, 174411 (2013).

[17] V. P. Amin and M. D. Stiles, Phys. Rev. B 94, 104420 (2016).

[18] L. Berger, Phys. Rev. B 2, 4559 (1970).

[19] J. Smit, Physica 24, 39 (1958).

[20] M. Dyakonov and V. Perel, Phys. Lett. A 35, 459 (1971).

[21] J. Sinova, D. Culcer, Q. Niu, N. A. Sinitsyn, T. Jungwirth, and A. H. MacDonald, Phys. Rev. Lett. 92, 126603 (2004).

[22] C. Gorini, U. Eckern, and R. Raimondi, Phys. Rev. Lett. 115, 076602 (2015).

[23] Y. Tian, L. Ye, and X. Jin, Phys. Rev. Lett. 103, 087206 (2009).

[24] M. Isasa, E. Villamor, L. E. Hueso, M. Gradhand, and F. Casanova, Phys. Rev. B 91, 024402 (2015).

[25] K.-W. Moon, J.-C. Lee, S.-B. Choe, and K.-H. Shin, Rev. Sci. Instrum. 80, 113904 (2009).

[26] E. C. Stoner and E. P. Wohlfarth, Philos. Trans. R. Soc. A 240, 599 (1948).

[27] U. H. Pi, K. W. Kim, J. Y. Bae, S. C. Lee, Y. J. Cho, K. S. Kim, and S. Seo, Appl. Phys. Lett. 97, 162507 (2010).

[28] See Supplemental Material at http://link.aps.org/supplemental/ 10.1103/PhysRevB.97.100405 for details on sample preparation, additional experimental data and details of the theory.

[29] F. D. Czeschka, L. Dreher, M. S. Brandt, M. Weiler, M. Althammer, I.-M. Imort, G. Reiss, A. Thomas, W. Schoch,
W. Limmer, H. Huebl, R. Gross, and S. T. B. Goennenwein, Phys. Rev. Lett. 107, 046601 (2011).

[30] R. L. Conte, G. V. Karnad, E. Martinez, K. Lee, N.-H. Kim, D.-S. Han, J.-S. Kim, S. Prenzel, T. Schulz, C.-Y. You, H. J. M. Swagten, and M. Kläui, AIP Adv. 7, 065317 (2017).

[31] X. Qiu, P. Deorani, K. Narayanapillai, K.-S. Lee, K.-J. Lee, H.-W. Lee, and H. Yang, Sci. Rep. 4, 4491 (2014).

[32] S.-M. Seo, K.-W. Kim, J. Ryu, H.-W. Lee, and K.-J. Lee, Appl. Phys. Lett. 101, 022405 (2012).

[33] A. V. Khvalkovskiy, V. Cros, D. Apalkov, V. Nikitin, M. Krounbi, K. A. Zvezdin, A. Anane, J. Grollier, and A. Fert, Phys. Rev. B 87, 020402 (2013).

[34] C. Kittel, Introduction to Solid State Physics, 8th ed. (Wiley, New York, 2005).

[35] T. Tanaka, H. Kontani, M. Naito, T. Naito, D. S. Hirashima, K. Yamada, and J. Inoue, Phys. Rev. B 77, 165117 (2008).

[36] G. Vignale, J. Supercond. Nov. Magn. 23, 3 (2010).

[37] A. Fert and P. M. Levy, Phys. Rev. Lett. 106, 157208 (2011).

[38] S. Lowitzer, M. Gradhand, D. Ködderitzsch, D. V. Fedorov, I. Mertig, and H. Ebert, Phys. Rev. Lett. 106, 056601 (2011).

[39] A. Hoffmann, IEEE Trans. Magn. 49, 5172 (2013).

[40] E. Sagasta, Y. Omori, M. Isasa, M. Gradhand, L. E. Hueso, Y. Niimi, Y. Otani, and F. Casanova, Phys. Rev. B 94, 060412(R) (2016).

[41] The fact that phonon skew scattering arises from anharmonic phonon-phonon processes suggests by analogy with the lattice thermal conductivity a nonmonotonic behavior at $T<T_{D}$. Note also that the current phonon skew scattering theory considers only leading-order three-phonon processes.

[42] A. Schmid, Z. Phys. 259, 421 (1973).

[43] G. R. Stewart, Rev. Sci. Instrum. 54, 1 (1983). 